\title{
Using Social Media to Characterize Public Sentiment Toward Medical Interventions Commonly Used for Cancer Screening: An Observational Study
}

Omar Metwally ${ }^{1,2}$, BS, MD; Seth Blumberg ${ }^{3,4}$, MD, PhD; Uri Ladabaum ${ }^{5}$, MD, MS; Sidhartha R Sinha ${ }^{5}$, MD

\footnotetext{
${ }_{1}^{1}$ Department of Clinical Informatics, University of California, San Francisco, San Francisco, CA, United States

${ }^{2}$ Department of Internal Medicine, Highland General Hospital, Oakland, CA, United States

${ }^{3}$ Francis I. Proctor Foundation, University of California, San Francisco, San Francisco, CA, United States

${ }^{4}$ St Mary's Medical Center, San Francisco, CA, United States

${ }^{5}$ School of Medicine, Division of Gastroenterology, Stanford University, Stanford, CA, United States
}

\section{Corresponding Author:}

Sidhartha R Sinha, MD

School of Medicine, Division of Gastroenterology

Stanford University

Alway Building, Room M211

300 Pasteur Drive

Stanford, CA, 94305-5187

United States

Phone: 16507236661

Fax: 16507235488

Email: sidsinha@stanford.edu

\section{Abstract}

Background: Although cancer screening reduces morbidity and mortality, millions of people worldwide remain unscreened. Social media provide a unique platform to understand public sentiment toward tools that are commonly used for cancer screening. Objective: The objective of our study was to examine public sentiment toward colonoscopy, mammography, and Pap smear and how this sentiment spreads by analyzing discourse on Twitter.

Methods: In this observational study, we classified 32,847 tweets (online postings on Twitter) related to colonoscopy, mammography, or Pap smears using a naive Bayes algorithm as containing positive, negative, or neutral sentiment. Additionally, we characterized the spread of sentiment on Twitter using an established model to study contagion.

Results: Colonoscopy-related tweets were more likely to express negative than positive sentiment (negative to positive ratio $1.65,95 \%$ CI $1.51-1.80, P<.001$ ), in contrast to the more positive sentiment expressed regarding mammography (negative to positive ratio $0.43,95 \%$ CI $0.39-0.47, P<.001)$. The proportions of negative versus positive tweets about Pap smear were not significantly different (negative to positive ratio $0.95,95 \%$ CI $0.87-1.04, P=.18$ ). Positive and negative tweets tended to share lexical features across screening modalities. Positive tweets expressed resonance with the benefits of early detection. Fear and pain were the principal lexical features seen in negative tweets. Negative sentiment for colonoscopy and mammography spread more than positive sentiment; no correlation with sentiment and spread was seen for Pap smear.

Conclusions: Analysis of social media data provides a unique, quantitative framework to better understand the public's perception of medical interventions that are commonly used for cancer screening. Given the growing use of social media, public health interventions to improve cancer screening should use the health perceptions of the population as expressed in social network postings about tests that are frequently used for cancer screening, as well as other people they may influence with such postings.

(J Med Internet Res 2017;19(6):e200) doi: 10.2196/jmir.7485

\section{KEYWORDS}

Twitter; sentiment analysis; cancer screening; colonoscopy; mammography; Pap smear; Papanicolaou test; social media; early detection of cancer 


\section{Introduction}

The US Preventive Services Task Force and numerous professional societies endorse colonoscopy, mammography, and Pap smear as effective screening modalities for colon, breast, and cervical cancer, respectively. Over 350,000 cases of these cancers are diagnosed yearly in the United States [1-5]. Despite the effectiveness of these and other screening modalities in reducing cancer-related mortality, millions of Americans do not undergo screening [6-13]. The reasons for this lack of adherence, particularly for these 3 tests, are multifold. Colonoscopy, mammography, and Pap smear are generally considered more invasive or involved than exclusively laboratory-based screening tests, such as those for high cholesterol or diabetes. Briefly, colonoscopy generally involves visualization via a flexible endoscope inserted into the rectum, and often biopsy, of colonic mucosa. To increase the chances of complete visualization of colonic mucosa, patients are typically advised to adhere to a restricted diet with avoidance of solid food the day prior to the procedure and are frequently required to drink large volumes of bowel-cleansing solutions that result in frequent bowel movements [14]. Mammography involves radiographic imaging after compression of the breast tissue, a process that can be uncomfortable for many women [15]. Pap smear entails insertion of a speculum into the vagina and use of a brush to scrape a sample of cervical cells, which allows the operator to examine cells microscopically for malignant and premalignant changes, but it can also be associated with pain and anxiety [16].

Patient questionnaires have identified discomfort, embarrassment, and various socioeconomic factors as barriers to participation in cancer screening $[4,17]$. However, studying patient perceptions of modalities commonly used for cancer screening using formal surveys is limited by several factors. First, these surveys can be costly to administer and do not provide real-time actionable information [18]. Second, monitoring the spread and changes in sentiment over time is limited by cost and diminishing response rates. Third, surveys ask specific questions and typically provide limited possible responses, which qualitatively and quantitatively limits the range of data generated by these interventions [19]. Infodemiology, which includes exploration of the distribution and determinants of information on the Internet to improve public health, provides an alternative method to study societal perceptions of health care, such as their sentiment regarding commonly used cancer screening interventions [20]. Infodemiologic studies have investigated numerous aspects of health, including attitudes toward and spread of illness as expressed on social media, search engines, and blogs; sentiment in chronic diseases; and the effectiveness of smoking cessation campaigns [20-24]. Analysis of social media postings offers a unique opportunity to overcome the limitations of conventional surveys and to understand core health care issues, such as why screening recommendations are often not followed. Among such networks, Twitter is relatively unique in that vast amounts of data are publicly available. Revealing differences in sentiment on social media toward various tools commonly used for screening and analyzing how

interventions to improve screening affect perception may lead to understanding how screening adherence can be increased.

In this study, we applied established methods in sentiment analysis and machine learning to Twitter data to characterize sentiment toward common interventions used for cancer screening. Similar methods have been applied to characterize patient attitudes toward various medical topics, including vaccines, illness, pain, and drug use [19,25-29]. We also quantified the way in which sentiment regarding interventions commonly used for cancer screening spreads on social networks, offering a unique opportunity to both understand health-related discourse propagation and gain insight on how to engineer outreach efforts more effectively.

\section{Methods}

\section{Recruitment}

We used the Twitter (Twitter, Inc) search application programming interface (API) to collect over 30,000 English-language tweets relating to colonoscopy $(10,262)$, mammography (12,002), and Pap smear (10,583) [12]. All tweets were collected on consecutive days over a 6-week period from December 2015 to January 2016. Colonoscopy-related tweets were identified by querying for the term "colonoscopy." Mammography-related tweets were identified by querying for the term "mammogram" or "mammography." Pap smear-related tweets were identified by querying for "pap smear," "pap test," "Papanicolaou test," "Papanicolaou AND screening," "pap AND cervical cancer," "pap AND pelvic exam," or "pap AND HPV." We obtained the data set by writing code to manually collect these publicly available tweets through the Twitter API, which is a sampling of up to $1 \%$ of the total number of tweets at any time (ie, the Twitter Firehose) [30-32]. The question of how representative the Twitter API is of the Firehose has been rigorously studied, and the limitations are discussed in the "Limitations" section below. Briefly, the quantity and quality of tweets delivered via the API depends on the keywords used to query the tweets, the user IDs specified, and geographic tags (if present). The Twitter API begins sampling using an undisclosed method once the $1 \%$ tweet threshold is reached. Following the precedent set by other Twitter-based studies, and according to our institutional review board's recommendations to exempt this study from review, we did not obtain consent from Twitter users, since we used the data in aggregate, and these data are publicly available. For each tweet, we recorded the content of the message and the number of retweets (how many times the tweet was propagated by other users).

\section{Sentiment Classification}

We classified sentiment in 2 separate steps as described in the literature [33,34]. First, 1500 tweets (500 for each screening modality) were manually labeled by an investigator as containing positive, negative, or neutral sentiment and were used to train the classifiers. For each screening modality, we trained a naive Bayes classifier, a classification algorithm in which training is based on prior probabilities with different variables assumed to be independent of one another a priori, to categorize all tweets as carrying either positive or nonpositive sentiment [35]. Then, we trained a second naive Bayes classifier 
to categorize all tweets as carrying either a negative sentiment or a nonnegative sentiment. Tweets that were positive and nonnegative were ultimately labeled as positive. Tweets that were negative and nonpositive were ultimately labeled as negative. Tweets that were classified as both nonpositive and nonnegative were grouped into a third, neutral category. No tweets were classified as both positive and negative.

\section{Validity}

To assess consistency in labeling, a random subset of the 1500 tweets were relabeled by the same investigator, with $96 \%$ concordance. To establish validity of our labeling system, a second investigator independently labeled tweets, with an interobserver concordance of $95 \%$. We characterized the accuracy of the classification algorithm by using 1200 of the labeled tweets as a training set and the remaining 300 as a testing set [36]. Our decision to use $75 \%$ of labeled tweets for training and $25 \%$ of labeled tweets for testing is consistent with validity assessment common in the machine learning literature; $20 \%$ to $33 \%$ of a labeled set is commonly used for testing purposes, with the remaining dataset used for training [36]. We inferred the true proportion of positive and negative tweets via a 2-step bootstrap method [37]. The first step of the bootstrap sampled individual classifications from the observed data with replacement. The second step labeled the bootstrapped classified data as positive, negative, or neutral based on contingency tables (Multimedia Appendix 1). To compute 2-sided $P$ values for the ratio of negative to positive sentiment, we applied a 1-step sample with replacement bootstrap to a null dataset that had the same number of observed neutral tweets, but an equal proportion of positive and negative tweets. The total size of the null dataset matched the observed data, and we assumed classification of the null datasets to be $100 \%$ accurate.

\section{Dissemination of Sentiment}

We analyzed word frequency in all original tweets for the most common words in positive and negative tweets. Demographic information about Twitter users was obtained from Demographics Pro (Demographics Pro Inc), a third-party tool providing inferred predictive analytics on demographic information about social media users with $95 \%$ or greater confidence based on multiple data sources $[26,38,39]$.

To assess the likelihood of a tweet to be propagated (ie, retweeted), we employed established concepts from the spread of infectious disease [40]. The effective reproduction number equals the expected secondary cases resulting from exposure to an infected individual $[41,42]$. Analogously, we defined the rate of propagation as the mean number of times a message is retweeted by a Twitter user. To account for heterogeneity of retweeting, we inferred rate of propagation by assigning a negative binomial distribution for the number of retweets each tweet generated. We determined the statistical difference of rate of propagation by Akaike information criterion score [41] and calculated corresponding $P$ values by chi-square modeling of the $\log$ likelihood ratio. $P$ values for the incidence of new tweets were determined based on assuming an underlying Poisson distribution for the introduction of new tweets.

\section{Results}

\section{Classifier Performance}

Our classifier labeled tweet sentiment with an accuracy of about $80 \%$. Importantly, no negatively classified tweets were manually labeled as positive, and only $4 \%$ of the positively classified tweets were manually labeled as negative (Multimedia Appendix 1). The misclassifications were predominantly for tweets with nonneutral sentiment classified as being neutral or for tweets with neutral sentiments being classified as nonneutral. As such, the overwhelming majority of misclassified tweets did not entail complete reversal of sentiment. One example of a tweet with neutral sentiment being classified as nonneutral (in this particular case, as negative) is "Worried about preparing for a colonoscopy? Don't. The preparation can be inconvenient, but it is not difficult or painful." Since we were using a naive Bayes classification algorithm, the most likely explanation for misclassification of this tweet is the presence of words with negative connotations, such as "painful" and "inconvenient." Similarly, this nonneutral (in this case, negative) tweet was incorrectly classified as neutral: "cant afford doctor just go to the airport. You get a free xray and breast exam. And if you mention Al Qaeda and you get a free colonoscopy."

\section{Differences in Sentiment Among Screening Modalities}

When adjusted for imperfections in classification, colonoscopy-related tweets were estimated to be 1.65 (95\% CI $1.51-1.80, P<.001)$ times more likely to express negative sentiment than positive sentiment (Figure 1 and Multimedia Appendix 2). In contrast, mammography-related tweets were 0.43 (95\% CI $0.39-0.47, P<.001$ ) times more likely to be negative than positive. The proportions of positive versus negative sentiment in Pap smear-related tweets were not significantly different (negative to positive ratio $0.95,95 \% \mathrm{CI}$ $0.87-1.04, P=.18$ ). The majority of tweets in all screening modalities were neutral.

\section{Demographic Analysis}

Table 1 provides aggregate sex and age information about Twitter users discussing each screening modality. A large proportion of Twitter users discussing an intervention commonly used for cancer screening were less than 45 years of age, generally younger than those who commonly pursue routine colon cancer screening (typically starting at age 50 years). In contrast to the demographics of the entire Twitter network, which is characterized by roughly equal proportions of male and female users, more male users commented on colonoscopy and, not surprisingly, more female users commented on mammography and Pap smear [43]. Interestingly, Twitter users commenting on colonoscopy, mammography, and Pap smear were younger than the average Twitter user. 
Figure 1. Sentiment expressed on Twitter regarding colonoscopy, mammography, and Pap smear (based on classification of over 30,000 tweets). A naive Bayes classifier was trained on labeled data and used to classify tweets relating to either colonoscopy, mammography, or Pap smear. Tests of statistical significance were undertaken using a bootstrap method with negative to positive sentiment ratio for colonoscopy $(1.65, P<.001)$, mammography $(0.43, P<.001)$, and Pap smear $(0.95, P=.18)$. The full results for the bootstrap analysis are shown in Multimedia Appendix 2.

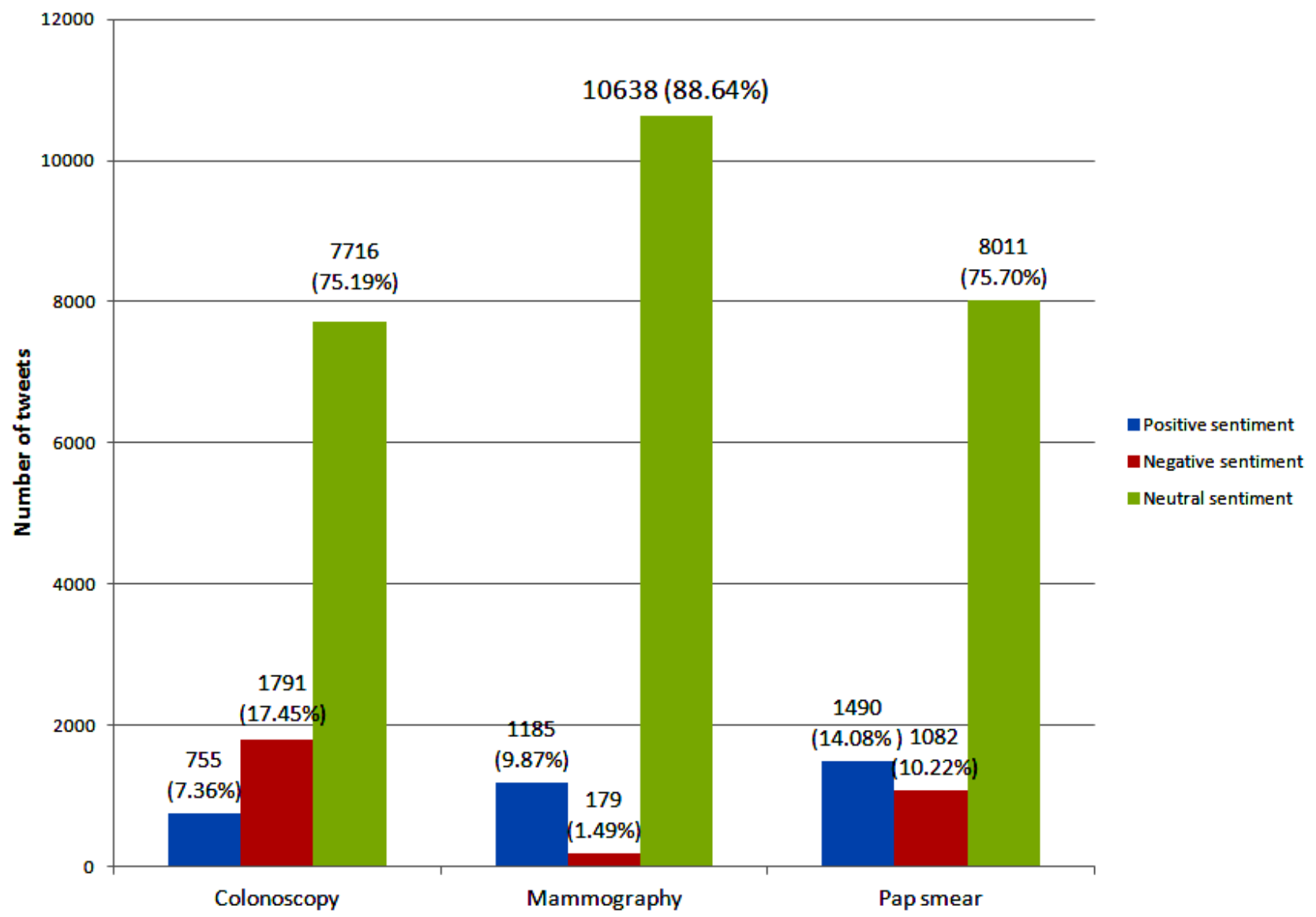

Table 1. Demographics ${ }^{\mathrm{a}}$ of users tweeting about cancer screening by screening modality $(\mathrm{N}=32,847)$.

\begin{tabular}{llll}
\hline Characteristics & Colonoscopy & Mammography & Pap smear \\
\hline Sex & & & $33.3 \%$ \\
$\quad$ Male & $56.7 \%$ & $36.4 \%$ & $66.7 \%$ \\
$\quad$ Female & $43.3 \%$ & $63.6 \%$ & \\
Age group (years) & & & $24.3 \%$ \\
$\leq 20$ & $18.4 \%$ & $10.9 \%$ & $34.3 \%$ \\
$21-29$ & $30.8 \%$ & $20.9 \%$ & $11.8 \%$ \\
$30-34$ & $15.9 \%$ & $14.7 \%$ & $18.6 \%$ \\
$35-44$ & $18.8 \%$ & $30.7 \%$ & $7.8 \%$ \\
$45-54$ & $10.1 \%$ & $15.5 \%$ & $2.2 \%$ \\
$55-64$ & $4.3 \%$ & $5.5 \%$ & $1.1 \%$ \\
$\geq 65$ & $1.7 \%$ & $1.5 \%$ & \\
\hline
\end{tabular}

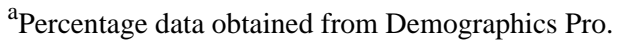

\section{Word Frequency Analysis}

Word frequency analysis for all 3 screening modalities demonstrated similarly perceived benefits of tools frequently used for cancer screening (Multimedia Appendix 3). Word frequency analysis showed that positively charged tweets most frequently contained words such as "health," "awareness," "screening," "detection," and "recommend." Negatively charged tweets most frequently contained words related to pain ("painful," "hurts"), anxiety associated with the procedure ("worried"), and issues with procedure preparation (“dehydration," "preparing"). Examination of individual tweets 
showed that positive sentiment was more likely to be expressed when providing information about a procedure or explaining the benefits of cancer screening (Table 2). Positive tweets might also provide a "call to action" to encourage friends or family to get screened. Negative tweets often expressed physical discomfort related to a screening modality and compared cancer screening to anxiety-provoking tasks or suggested a level of social inappropriateness with the topic. Tweets with negative sentiment often included sarcastic humor.

Table 2. Examples of positive and negative tweets.

\begin{tabular}{lll}
\hline $\begin{array}{l}\text { Type of tweet and } \\
\text { modality }\end{array}$ & Positive sentiment & Negative sentiment
\end{tabular}

\section{Information on the procedure (positive) or fear of pain or harm (negative)}

Colonoscopy

Worried about preparing for a colonoscopy? Don't. The preparation can be inconvenient, but it is not difficult or painful.

Mammography For women with dense breasts, ultrasound could help diagnose breast cancer.

Pap smear Getting a \#Paptest is one of the best things you can do to prevent \#cervicalcancer.
I don't think my breast have ever been so smashed and squeezed. And here I thought a mammogram would be like taking an X-ray--NOPE!

Corsets $=$ struggling to breathe while getting a constant mammogram.

Getting a colonoscopy can cause the patient to explode (methane+oxygen+electrical spark).

So my mom never had a pap smear until after she had me, when she was 37 . I grew up hearing about how horrific it was. It really hurt her.

\section{Benefits of cancer screening (positive) or "I'd rather" tweets (negative)}

Colonoscopy

Call to Action (positive) or Other (negative)

Colonoscopy

Have a friend turning 50? Encourage them to get their colonoscopy; it could save their life.

Mammography Ladies get that mammogram because it saved my little sister from a very aggressive breast cancer. Make it a XMas present to yourself.

Pap smear I I went for my first ever Pap Test today *feeling proud \& brave*. Thanks to all the lesbian women who urged/reminded me to go! Hello ladies schedule your mammogram today. Include health in your new year's resolution.
Things I would rather do than my exams: re-organise a forever 21 store, eat my own vomit, peel 4,000 potatoes with a spork, colonoscopy prep

I can think of a few better places: Gates of Hell, during a colonoscopy, Mordor, a Joel Osteen Conference..

i'd rather have a mammogram done while being awake as they remove my kidney

I would rather give myself a pap smear in the middle of Macy's than read your Christmas newsletter, Aunt Karen

i would hate to get a colonoscopy...

My first colonoscopy will be done by a coroner at my autopsy

Today a colleague told me that he's having a colonoscopy this week. I need a new job.

How a mammogram actually causes breast cancer.

Your mothers so dumb she went to Dr. Dre for a pap smear
Colonoscopies are not just simple, harmless tests. Here are the pros and cons to consider.

Is This Why Mammogram Recommendations Have Changed?

For me, a Pap Test just ended up being an unexpected trigger. For various reasons, some of which I will never know. 
Figure 2. The fraction of original tweets and the rate of propagation for each modality. Upward (downward) pointing triangles represent positive (negative) tweets. Lines are for visualization purposes only. The difference in rate of propagation between positive and negative tweets was significant for colonoscopy $(P=.001)$ and mammography $(P=.02)$ but not for Pap smear $(P=.83)$.

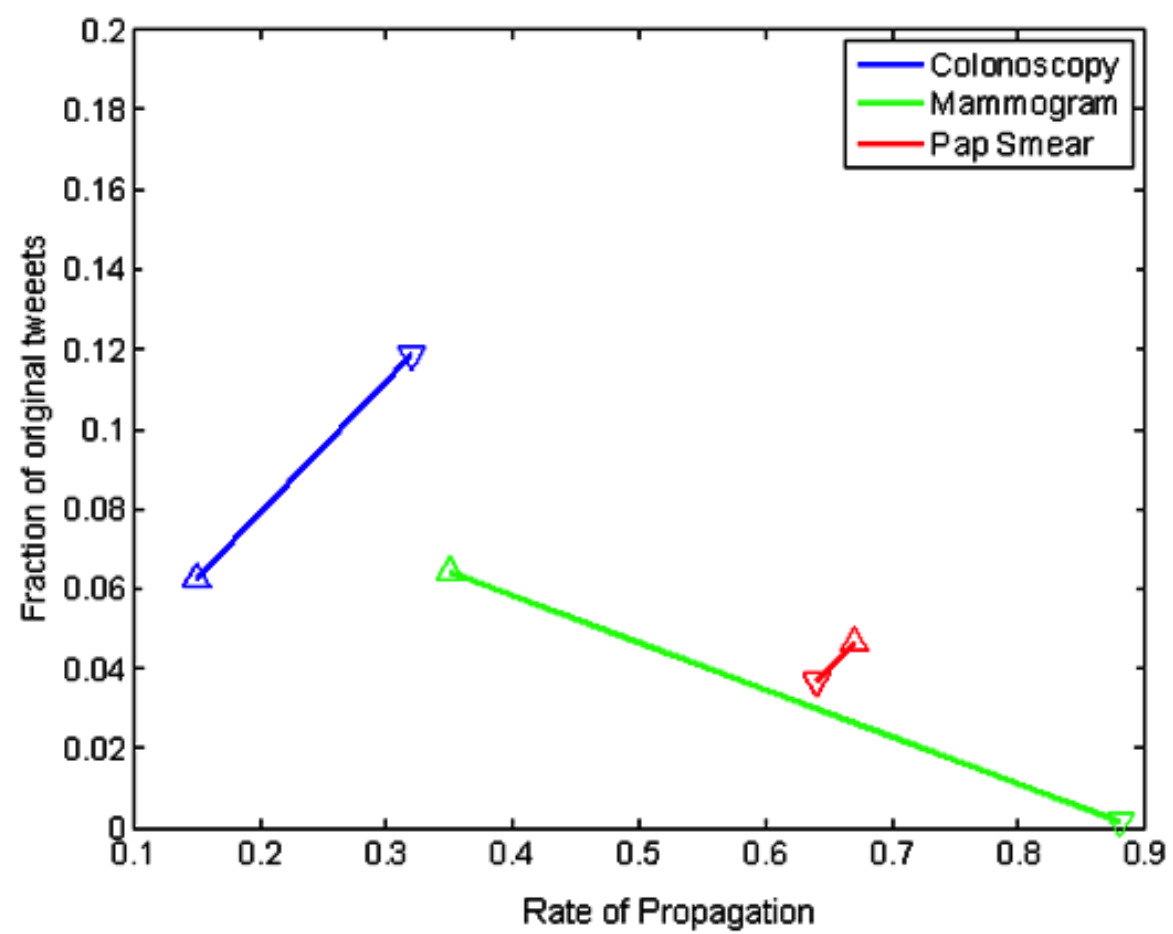

\section{Propagation of Sentiment}

The proportion of tweets with positive versus negative sentiment is dependent on both the frequency of new tweets and the rate at which these new tweets are retweeted (Figure 2 and Multimedia Appendix 4) [44]. Comparison of positively versus negatively classified tweets showed that, for colonoscopy, negative sentiment both was more frequent in original tweets and spread more than positive ones. New tweets regarding mammography were typically positive rather than negative. However, the few negative-sentiment tweets toward mammography spread much more than those with positive sentiment. For Pap smear, negative and positive tweets had similar characteristics with regard to their spread and the frequency of new tweets.

\section{Discussion}

\section{Principal Results}

By using automated sentiment classifiers, we were able to analyze much larger bodies of data than in typical patient survey-based studies. Understanding basic differences in sentiment between interventions commonly used for screening, such as the greater prevalence of negative sentiment regarding colonoscopy compared with mammography, could lead to more targeted, effective interventions, as well as the real-time means to assess the effects of such interventions. Such comparisons could, for example, foster organizations promoting screening to learn from each other to more effectively maintain social media interventions to promote positive sentiment for these lifesaving medical interventions. Beyond sentiment analysis, word frequency analyses can provide quantitative as well as qualitative insight into potential reasons for differences in sentiment and can identify areas on which to focus education efforts. For instance, pain and fear were common themes in negative tweets about all 3 modalities, findings that have been echoed, at times inconsistently, by formal patient surveys [17].

\section{Comparison With Prior Work}

We employed novel machine learning algorithms to understand sentiment on social media regarding tools commonly used for screening. Understanding opinions regarding these changes by analyzing social media could be valuable in assessing health policy changes and implementing new policies. With respect to cancer screening, public reactions to changes in screening recommendations from professional societies could also be monitored, and false perceptions could be addressed immediately.

\section{Limitations}

Despite the large number of public tweets available for analysis, this is an imperfect representation of the population at large. The age of Twitter users sampled is generally younger than the target screening population, who commonly use the medical tests investigated in this study, limiting the generalizability of our results to older populations. However, this limitation also provides useful insight. For example, one hypothesis to explain the negative sentiments regarding colonoscopy in younger users is that some of these persons may have conditions such as inflammatory bowel disease and require invasive, potentially embarrassing interventions that their peers do not require. It is unclear how younger persons' sentiments toward colonoscopy might affect the sentiments of older persons, including the relatives of the tweet authors, who may be eligible for 
age-appropriate colon cancer screening. While references to colonoscopy on Twitter may not entirely reflect screening and surveillance, these are the most common indications for colonoscopy [13]. The same is true for mammography and Pap smear. Yet even understanding perceptions of a tool commonly used for screening is telling. This information still represents public opinion, albeit a younger population, and understanding the perceptions of this younger population may influence future screening decisions as well. Influencing younger users may in fact be a strategy to further improve cancer screening adherence. The relatively small subgroup sample sizes limited the demographic analysis possible through Demographics Pro. Additionally, potentially vulnerable groups, including minorities at risk for poor preventive health use, may not be represented. Nonetheless, we believe that public expressions of sentiment provide insight that may not necessarily be reflected by formal surveys into how the screening modalities that we studied are perceived by the public. We chose to capture whether tweets mentioning tools commonly used for cancer screening were generally positive or negative versus attempting to discern whether the sentiment was expressed specifically regarding the actual screening procedure itself. More nuanced sentiment analysis methods capable of discerning meaning by analyzing sentences as aggregates of phrases and their modifiers may improve our understanding of public discourse specifically related to cancer screening [45]. We believe that even capturing such nonscreening-related mentions of these interventions provides valuable insight into public opinion for these tools used by millions to improve health.

How well the Twitter API samples the total corpus of tweets (the Twitter Firehose) has been studied by Morstatter et al and has yielded heterogeneous results [32]. The API's sampling is imperfect and depends to a large extent on the type of analysis undertaken. For example, those authors found that the $1 \%$ sampling becomes substantially more representative when tweets are collected over consecutive days, as was the case in our study. The quality of the API's sampling decreases when the number of hashtags or query keywords decreases, which would theoretically affect the quality of sampled colonoscopy tweets (where "colonoscopy" is the only queried term) more than Pap smear-related tweets (which we sampled using 10 unique keywords). Correcting for this sampling bias is difficult given that Twitter does not disclose how sampling is performed, but it should be acknowledged in infodemiologic studies that use the Twitter search API.

\section{Conclusion}

We have analyzed tweets about interventions commonly used for cancer screening to assess public sentiment about these interventions. There were substantially more negative than positive tweets about colonoscopy, but not mammography or Pap smear. Tweet propagation in the social network was greater for negative than for positive tweets about colonoscopy and mammography, suggesting a possible disproportionate impact of negative sentiment for these screening tests. Examination of large data sets available from the Twitter social network using automated algorithms provides an opportunity to examine public attitudes toward cancer screening and other health care interventions that might lead to policy changes, novel programs, and more refined counseling guidelines that improve public attitudes and health-related behaviors.

\section{Acknowledgments}

Seth Blumberg was supported by the RAPIDD Program of the Science and Technology Directorate, US Department of Homeland Security (Research and Policy for Infectious Disease Dynamics). Sidhartha Sinha was supported by a KL2 Mentored Career Development Award of the Stanford Clinical and Translational Science Award to Spectrum (National Institutes of Health KL2 TR 001083) and the Division of Gastroenterology and Hepatology at Stanford University School of Medicine, Stanford, CA, USA. We are grateful to Demographics Pro for providing us with demographic information. The Python code we used to implement the naive Bayes classification algorithm was built on code from the public domain, and the authors are acknowledged in the source code we provide [46].

\section{Authors' Contributions}

OM contributed to study design, conceptualization, methodology, software, validation, formal analysis, data curation, writing of the original draft, review, and editing, and visualization. SB contributed to study design, methodology, software, validation, formal analysis, data curation, writing review and editing, and visualization. UL contributed to study design, writing review and editing, and formal analysis. SRS contributed to study design, conceptualization, validation, formal analysis, data curation, writing of the original draft, review, and editing, and visualization.

\section{Conflicts of Interest}

None declared.

\section{Multimedia Appendix 1}

Contingency tables for labeling bootstrapped classified data.

[PDF File (Adobe PDF File), 26KB-Multimedia Appendix 1] 


\section{Multimedia Appendix 2}

Tweet sentiment classification with cancer screen modality.

[PDF File (Adobe PDF File), 27KB-Multimedia Appendix 2]

\section{Multimedia Appendix 3}

Word frequency analysis of common themes.

[PDF File (Adobe PDF File), 24KB-Multimedia Appendix 3]

\section{Multimedia Appendix 4}

Rate of propagation for positive and negative tweets.

[PDF File (Adobe PDF File), 59KB-Multimedia Appendix 4]

\section{References}

1. Nishihara R, Wu K, Lochhead P, Morikawa T, Liao X, Qian ZR, et al. Long-term colorectal-cancer incidence and mortality after lower endoscopy. N Engl J Med 2013 Sep 19;369(12):1095-1105 [FREE Full text] [doi: 10.1056/NEJMoa1301969] [Medline: 24047059]

2. Levin B, Lieberman DA, McFarland B, Andrews KS, Brooks D, Bond J, American Cancer Society Colorectal Cancer Advisory Group, US Multi-Society Task Force, American College of Radiology Colon Cancer Committee. Screening and surveillance for the early detection of colorectal cancer and adenomatous polyps, 2008: a joint guideline from the American Cancer Society, the US Multi-Society Task Force on Colorectal Cancer, and the American College of Radiology. Gastroenterology 2008 May;134(5):1570-1595. [doi: 10.1053/j.gastro.2008.02.002] [Medline: 18384785]

3. Inadomi JM, Vijan S, Janz NK, Fagerlin A, Thomas JP, Lin YV, et al. Adherence to colorectal cancer screening: a randomized clinical trial of competing strategies. Arch Intern Med 2012 Apr 9;172(7):575-582 [FREE Full text] [doi:

10.1001/archinternmed.2012.332] [Medline: 22493463]

4. Wee CC, McCarthy EP, Phillips RS. Factors associated with colon cancer screening: the role of patient factors and physician counseling. Prev Med 2005 Jul;41(1):23-29. [doi: 10.1016/j.ypmed.2004.11.004] [Medline: 15916989]

5. Centers for Disease Control and Prevention. United States cancer statistics: 1999-2012 incidence and mortality data. Atlanta, GA: U.S. Department of Health and Human Services, Centers for Disease Control and Prevention and National Cancer Institute; 2015. URL: http://www.cdc.gov/uscs [accessed 2015-12-14] [WebCite Cache ID 6peu3g5B1]

6. American Cancer Society. What are the key statistics about cervical cancer?. 2016. URL: https://www.cancer.org/cancer/ cervical-cancer/about/key-statistics.html [accessed 2017-04-12] [WebCite Cache ID 6peuBzo3G]

7. U.S. Preventive Services Task Force. Final summary: cervical cancer: screening. Rockville, MD: U.S. Preventive Services Task Force; 2012. URL: http://www.uspreventiveservicestaskforce.org/Page/Document/UpdateSummaryFinal/ cervical-cancer-screening [accessed 2016-02-08] [WebCite Cache ID 6peuKAWoA]

8. Surveillance, Epidemiology, and End Results (SEER) Program. Cancer stat facts: female breast cancer. Rockville, MD: U.S. Department of Health and Human Services, National Institutes of Health, National Cancer Institute; 2017. URL: https:/ /seer.cancer.gov/statfacts/html/breast.html [accessed 2017-05-29] [WebCite Cache ID 6qpBoDveX]

9. Paci E. Summary of the evidence of breast cancer service screening outcomes in Europe and first estimate of the benefit and harm balance sheet. J Med Screen 2012;19 Suppl 1:5-13. [doi: 10.1258/jms.2012.012077] [Medline: 22972806]

10. Ferrante JM, Chen P, Crabtree BF, Wartenberg D. Cancer screening in women: body mass index and adherence to physician recommendations. Am J Prev Med 2007 Jun;32(6):525-531 [FREE Full text] [doi: 10.1016/j.amepre.2007.02.004] [Medline: 17533069]

11. Koong S, Yen AM, Chen TH. Efficacy and cost-effectiveness of nationwide cervical cancer screening in Taiwan. J Med Screen 2006;13 Suppl 1:S44-S47. [Medline: 17227642]

12. Arroyave AM, Penaranda EK, Lewis CL. Organizational change: a way to increase colon, breast and cervical cancer screening in primary care practices. J Community Health 2011 Apr;36(2):281-288. [doi: 10.1007/s10900-010-9309-7] [Medline: 20835777]

13. Ladabaum U, Levin Z, Mannalithara A, Brill JV, Bundorf MK. Colorectal testing utilization and payments in a large cohort of commercially insured US adults. Am J Gastroenterol 2014 Oct;109(10):1513-1525. [doi: 10.1038/ajg.2014.64] [Medline: 24980877]

14. Inadomi JM. Screening for colorectal neoplasia. N Engl J Med 2017 Dec 20;376(16):1599-1600. [doi: 10.1056/NEJMc1702535] [Medline: 28423294]

15. Domar AD, Eyvazzadeh A, Allen S, Roman K, Wolf R, Orav J, et al. Relaxation techniques for reducing pain and anxiety during screening mammography. AJR Am J Roentgenol 2005 Feb;184(2):445-447. [doi: 10.2214/ajr.184.2.01840445] [Medline: $\underline{15671361]}$ 
16. Basen-Engquist K, Shinn EH, Warneke C, de Moor C, Le T, Richards-Kortum R, et al. Patient distress and satisfaction with optical spectroscopy in cervical dysplasia detection. Am J Obstet Gynecol 2003 Oct;189(4):1136-1142. [Medline: 14586367]

17. Ghanouni A, Plumb A, Hewitson P, Nickerson C, Rees CJ, von Wagner C. Patients' experience of colonoscopy in the English Bowel Cancer Screening Programme. Endoscopy 2016 Mar;48(3):232-240. [doi: 10.1055/s-0042-100613] [Medline: 26841268]

18. Alemi F, Torii M, Clementz L, Aron DC. Feasibility of real-time satisfaction surveys through automated analysis of patients' unstructured comments and sentiments. Qual Manag Health Care 2012;21(1):9-19. [doi: 10.1097/QMH.0b013e3182417fc4] [Medline: 22207014]

19. Greaves F, Ramirez-Cano D, Millett C, Darzi A, Donaldson L. Use of sentiment analysis for capturing patient experience from free-text comments posted online. J Med Internet Res 2013;15(11):e239 [FREE Full text] [doi: 10.2196/jmir.2721] [Medline: 24184993]

20. Eysenbach G. Infodemiology and infoveillance: framework for an emerging set of public health informatics methods to analyze search, communication and publication behavior on the Internet. J Med Internet Res 2009;11(1):e11 [FREE Full text] [doi: 10.2196/jmir.1157] [Medline: 19329408]

21. Eysenbach G. Infodemiology: The epidemiology of (mis)information. Am J Med 2002 Dec 15;113(9):763-765. [Medline: 12517369]

22. Eysenbach G. Websites on screening for breast cancer: "infodemiology" studies have surely had their day. BMJ 2004 Mar 27;328(7442):769; author reply 769-769; author reply 770 [FREE Full text] [doi: 10.1136/bmj.328.7442.769-b] [Medline: 15044306]

23. Delir HP, Kang Y, Buchbinder R, Burstein F, Whittle S. Investigating subjective experience and the influence of weather among individuals with fibromyalgia: a content analysis of Twitter. JMIR Public Health Surveill 2017 Jan 19;3(1):e4 [FREE Full text] [doi: 10.2196/publichealth.6344] [Medline: 28104577]

24. Chew C, Eysenbach G. Pandemics in the age of Twitter: content analysis of tweets during the 2009 H1N1 outbreak. PLoS One 2010;5(11):e14118 [FREE Full text] [doi: 10.1371/journal.pone.0014118] [Medline: 21124761]

25. Tighe PJ, Goldsmith RC, Gravenstein M, Bernard HR, Fillingim RB. The painful tweet: text, sentiment, and community structure analyses of tweets pertaining to pain. J Med Internet Res 2015;17(4):e84 [FREE Full text] [doi: 10.2196/jmir.3769] [Medline: 25843553]

26. Cavazos-Rehg PA, Krauss M, Fisher SL, Salyer P, Grucza RA, Bierut LJ. Twitter chatter about marijuana. J Adolesc Health 2015 Feb;56(2):139-145. [doi: 10.1016/j.jadohealth.2014.10.270] [Medline: 25620299]

27. Daniulaityte R, Chen L, Lamy FR, Carlson RG, Thirunarayan K, Sheth A. "When 'bad' is 'good'": identifying personal communication and sentiment in drug-related tweets. JMIR Public Health Surveill 2016 Oct 24;2(2):e162 [FREE Full text] [doi: 10.2196/publichealth.6327] [Medline: 27777215]

28. Massey PM, Leader A, Yom-Tov E, Budenz A, Fisher K, Klassen AC. Applying multiple data collection tools to quantify human papillomavirus vaccine communication on Twitter. J Med Internet Res 2016 Dec 05;18(12):e318 [FREE Full text] [doi: 10.2196/jmir.6670] [Medline: 27919863]

29. Rose SW, Jo CL, Binns S, Buenger M, Emery S, Ribisl KM. Perceptions of menthol cigarettes among Twitter users: content and sentiment analysis. J Med Internet Res 2017 Feb 27;19(2):e56 [FREE Full text] [doi: 10.2196/jmir.5694] [Medline: 28242592]

30. Twitter developers documentation: the search API. San Francisco, CA: Twitter Inc; 2017. URL: https://dev.twitter.com/ rest/public/search [accessed 2015-02-17] [WebCite Cache ID 6qJGWK7Zu]

31. Gnip. Boulder, CO: Gnip, Inc; 2016. URL: https://gnip.com/ [accessed 2017-04-12] [WebCite Cache ID 6peuTPZB7]

32. Morstatter F, Pfeffer J, Liu H, Carley K. Is the sample good enough? Comparing data from Twitter's streaming API with Twitter's firehose. Palo Alto, CA: AAAI Press; 2013 Presented at: 7th International AAAI Conference on Weblogs and Social Media; July 8-11, 2013; Cambridge, MA, USA p. 400-408 URL: https://arxiv.org/pdf/1306.5204.pdf

33. Natural language toolkit.: NLTK Project; 2017 Jan 02. URL: http://www.nltk.org/ [accessed 2017-04-12] [WebCite Cache ID 6peuVeLOE]

34. Ranco G, Aleksovski D, Caldarelli G, Grčar M, Mozetič I. The effects of Twitter sentiment on stock price returns. PLoS One 2015;10(9):e0138441 [FREE Full text] [doi: 10.1371/journal.pone.0138441] [Medline: 26390434]

35. Manning C, Raghavan P, Schuze H. Introduction to Information Retrieval. Cambridge, UK: Cambridge University Press; 2008.

36. Pagolu V, Challa K, Panda G, Majhi B. Sentiment analysis of Twitter data for predicting stock market movements. 2016 Oct 03 Presented at: International Conference on Signal Processing, Communication, Power and Embedded Systems. SCOPES 2016; Oct 3-5, 2016; Paralakhemundi, Odisha, India p. 3-5 URL: https://arxiv.org/pdf/1610.09225.pdf

37. Efron B. Bootstrap methods: another look at the jackknife. Ann Stat 1979 Jan;7(1):1-26. [doi: 10.1214/aos/1176344552]

38. Cavazos-Rehg P, Krauss M, Grucza R, Bierut L. Characterizing the followers and tweets of a marijuana-focused Twitter handle. J Med Internet Res 2014;16(6):e157 [FREE Full text] [doi: 10.2196/jmir.3247] [Medline: 24974893]

39. Understand and grow your social audience. Carrboro, NC: Demographics Pro Inc URL: http://www.demographicspro.com/ [accessed 2017-04-12] [WebCite Cache ID 6peuZYwPn] 
40. Corley CD, Mihalcea R, Mikler AR, Sanfilippo AP. Predicting individual affect of health interventions to reduce HPV prevalence. Adv Exp Med Biol 2011;696:181-190. [doi: 10.1007/978-1-4419-7046-6 18] [Medline: 21431558]

41. Blumberg S, Funk S, Pulliam JR. Detecting differential transmissibilities that affect the size of self-limited outbreaks. PLoS Pathog 2014 Oct;10(10):e1004452 [FREE Full text] [doi: 10.1371/journal.ppat.1004452] [Medline: 25356657]

42. Blumberg S, Lloyd-Smith JO. Inference of R(0) and transmission heterogeneity from the size distribution of stuttering chains. PLoS Comput Biol 2013;9(5):e1002993 [FREE Full text] [doi: 10.1371/journal.pcbi.1002993] [Medline: 23658504]

43. Greenwood S, Perrin A, Duggan M. Social media update 2016. Washington, DC: Pew Research Center; 2016 Nov 11. URL: http://www.pewinternet.org/2016/11/11/social-media-update-2016/ [accessed 2017-04-12] [WebCite Cache ID 6peufALgU]

44. Blumberg S, Enanoria WT, Lloyd-Smith JO, Lietman TM, Porco TC. Identifying postelimination trends for the introduction and transmissibility of measles in the United States. Am J Epidemiol 2014 Jun 01;179(11):1375-1382 [FREE Full text] [doi: 10.1093/aje/kwu068] [Medline: 24786800]

45. Socher R, Perelygin A, Wu J, Chuang J, Manning C, Ng A, et al. Recursive deep models for semantic compositionality over a sentiment treebank. 2013 Presented at: Conference on Empirical Methods in Natural Language Processing; Oct 18-21, 2013; Seattle, WA, USA.

46. Metwally O. Bitbucket. Overview. Sydney, Australia: Atlassian; 2017. URL: https://bitbucket.org/osmode/cancer_screening/ [accessed 2017-06-02] [WebCite Cache ID 6qvESTouQ]

\section{Abbreviations}

API: application programming interface

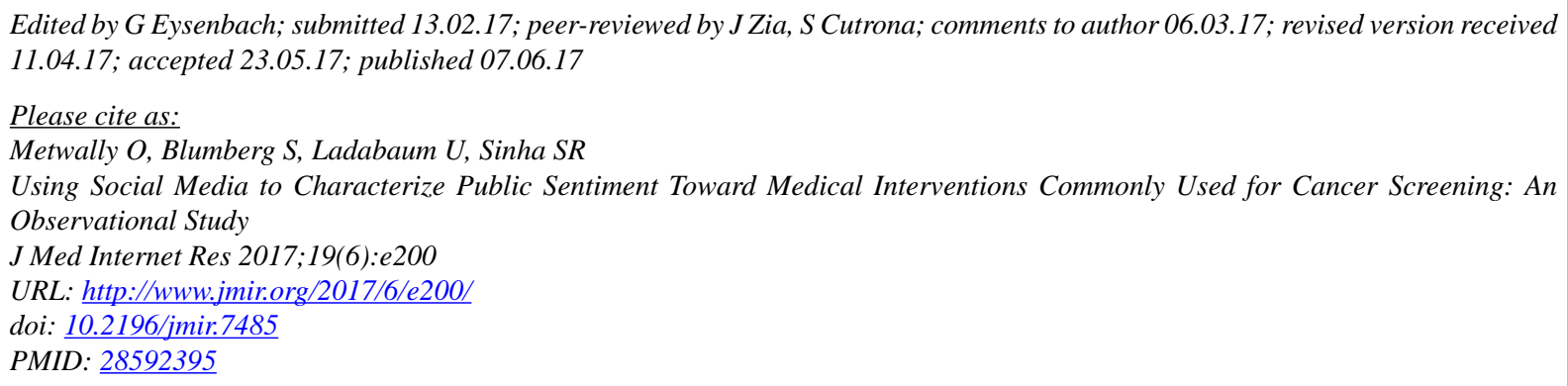

(C) Omar Metwally, Seth Blumberg, Uri Ladabaum, Sidhartha R. Sinha. Originally published in the Journal of Medical Internet Research (http://www.jmir.org), 07.06.2017. This is an open-access article distributed under the terms of the Creative Commons Attribution License (https://creativecommons.org/licenses/by/4.0/), which permits unrestricted use, distribution, and reproduction in any medium, provided the original work, first published in the Journal of Medical Internet Research, is properly cited. The complete bibliographic information, a link to the original publication on http://www.jmir.org/, as well as this copyright and license information must be included. 